\title{
REMARKS ON POISSON REDUCTION ON $K$-SYMPLECTIC MANIFOLDS
}

\author{
ADARA M. BLAGA
}

Communicated by Charles-Michel Marle

\begin{abstract}
Two reduced standard $k$-symplectic Poisson manifolds with respect to the action of a Lie group $G$ are considered, and the relation between the corresponding Poisson structures is established.
\end{abstract}

\section{Introduction}

Starting with a Poisson manifold, one can construct by reduction other Poisson manifolds. This procedure is well known and important in the symplectic mechanics having applications in fluids [5], electromagnetism and plasma physics [4], etc. Let us mention also that Juan-Pablo Ortega and Tudor Raţiu [7] described the Poisson reduction specifying the assumptions under that a Poisson manifold could be reduced to a Poisson manifold, too.

In what follows we shall present the Poisson reduction in the case of the standard $k$-symplectic manifold $\left(T_{k}^{1}\right)^{*} \mathbb{R}^{n}$ with the canonical $k$-symplectic structure induced from $\left(\mathbb{R}^{n}, \omega_{0}\right)$ [1]. Then, using a diffeomorphism, we can endow $T_{k}^{1} \mathbb{R}^{n}$ with a $k$-symplectic structure that will be reduced, too (the two manifolds $T_{k}^{1} \mathbb{R}^{n}=$ $T \mathbb{R}^{n} \oplus k \oplus \oplus \mathbb{R}^{n}$ and respectively $\left(T_{k}^{1}\right)^{*} \mathbb{R}^{n}=T^{*} \mathbb{R}^{n} \oplus k \oplus \oplus T^{*} \mathbb{R}^{n}$ will be identified with the Whitney sum of $k$-copies of $T \mathbb{R}^{n}$ and respectively of $T^{*} \mathbb{R}^{n}[6]$ ). Finally, we shall discuss the relation between the two induced Poisson structures on the reduced manifolds.

In order to do this, we consider an appropriate action of a Lie group $G$ on the two manifolds. Such canonical actions can be obtained, for instance, by lifting an arbitrary action of $G$ on $\mathbb{R}^{n}$ to $\left(T_{k}^{1}\right)^{*} \mathbb{R}^{n}$ and $T_{k}^{1} \mathbb{R}^{n}$ respectively. 


\section{Poisson Reduction}

Recall that a $k$-symplectic manifold $\left(M, \omega_{i}, V\right)_{1 \leq i \leq k}[1]$ is an $(n+n k)$-dimensional smooth manifold $M$ together with $k$ two-forms $\omega_{i}, 1 \leq i \leq k$, and an $n k$-dimensional distribution $V$ that satisfy the conditions

1. $\omega_{i}$ is closed, for every $1 \leq i \leq k$

2. $\bigcap_{i=1}^{k} \operatorname{ker} \omega_{i}=\{0\}$

3. $\omega_{\left.i\right|_{V \times V}}=0$, for every $1 \leq i \leq k$.

The canonical model for this structure is the standard $k$-symplectic manifold $\left(\left(T_{k}^{1}\right)^{*} \mathbb{R}^{n}, \omega_{i}, V\right)_{1 \leq i \leq k}$ [3], where $\omega_{i}, 1 \leq i \leq k$ are the differentials of the Liouville one-forms and $V$ is the vertical distribution. In Darboux coordinates $\left(q^{\alpha}, p_{\alpha}^{i}\right)_{1 \leq i \leq k, 1 \leq \alpha \leq n}$, we have

1. $\omega_{i}=\sum_{\alpha=1}^{n} \mathrm{~d} q^{\alpha} \wedge \mathrm{d} p_{\alpha}^{i}, 1 \leq i \leq k$

2. $V=<\frac{\partial}{\partial p_{\alpha}^{i}}>_{1 \leq i \leq k, 1 \leq \alpha \leq n}$.

Consider an action $\Phi: G \times \mathbb{R}^{n} \rightarrow \mathbb{R}^{n}$ of a Lie group $G$ on $\mathbb{R}^{n}$ and define the lifted action $\Phi^{T_{k}^{*}}: G \times\left(T_{k}^{1}\right)^{*} \mathbb{R}^{n} \rightarrow\left(T_{k}^{1}\right)^{*} \mathbb{R}^{n}$ to the standard $k$-symplectic manifold $\left(T_{k}^{1}\right)^{*} \mathbb{R}^{n}$

$$
\begin{aligned}
& \Phi^{T_{k}^{*}}: G \times\left(T_{k}^{1}\right)^{*} \mathbb{R}^{n} \rightarrow\left(T_{k}^{1}\right)^{*} \mathbb{R}^{n} \\
& \Phi^{T_{k}^{*}}\left(g, \alpha_{1 q}, \ldots, \alpha_{k q}\right):=\left(\alpha_{1 q} \circ\left(\Phi_{g^{-1}}\right)_{* \Phi_{g}(q)}, \ldots, \alpha_{k q} \circ\left(\Phi_{g^{-1}}\right)_{* \Phi_{g}(q)}\right)
\end{aligned}
$$

where $g \in G,\left(\alpha_{1}, \ldots, \alpha_{k}\right) \in\left(T_{k}^{1}\right)^{*} \mathbb{R}^{n}, q \in \mathbb{R}^{n}$.

This action is $k$-symplectic [6], that is, it preserves the standard $k$-symplectic structure $\omega_{1}, \ldots, \omega_{k}$ on $\left(T_{k}^{1}\right)^{*} \mathbb{R}^{n}$. Moreover, the Liouville one-forms $\theta_{i}, 1 \leq i \leq k$ $\left(\omega_{i}:=\mathrm{d} \theta_{i}, 1 \leq i \leq k\right)$ are $G$-invariant, that is

$$
\left(\Phi_{g}^{T_{k}^{*}}\right)^{*} \theta_{i}=\theta_{i}, \quad g \in G, \quad 1 \leq i \leq k .
$$

Similarly one can lift the action $\Phi$ to $T_{k}^{1} \mathbb{R}^{n}$

$$
\begin{gathered}
\Phi^{T_{k}}: G \times T_{k}^{1} \mathbb{R}^{n} \rightarrow T_{k}^{1} \mathbb{R}^{n} \\
\Phi^{T_{k}}\left(g, v_{1 q}, \ldots, v_{k q}\right):=\left(\left(\Phi_{g}\right)_{* q} v_{1 q}, \ldots,\left(\Phi_{g}\right)_{* q} v_{k q}\right)
\end{gathered}
$$


for $g \in G,\left(v_{1}, \ldots, v_{k}\right) \in T_{k}^{1} \mathbb{R}^{n}, q \in \mathbb{R}^{n}$.

Using a diffeomorphism $F: T_{k}^{1} \mathbb{R}^{n} \rightarrow\left(T_{k}^{1}\right)^{*} \mathbb{R}^{n}$, equivariant with respect to these actions, that is $\Phi_{g}^{T_{k}^{*}} \circ F=F \circ \Phi_{g}^{T_{k}}$, for any $g \in G$, we can take the pull-back on $T_{k}^{1} \mathbb{R}^{n}$ of the $k$-symplectic structure $\left(\omega_{i}, V\right)_{1 \leq i \leq k}$ on the standard $k$-symplectic manifold $\left(T_{k}^{1}\right)^{*} \mathbb{R}^{n}[3]$, and define $\left(\left(\omega_{F}\right)_{i}, V_{F}\right)_{1 \leq i \leq k}$ by

1. $\left(\omega_{F}\right)_{i}=F^{*} \omega_{i}$

2. $V_{F}=\operatorname{ker}\left(\pi_{F}\right)_{*}$

for any $1 \leq i \leq k$, where $\pi_{F}: T_{k}^{1} \mathbb{R}^{n} \rightarrow \mathbb{R}^{n}, \pi_{F}\left(v_{1 q}, \ldots, v_{k q}\right):=q$. Then $\left(T_{k}^{1} \mathbb{R}^{n},\left(\omega_{F}\right)_{i}, V_{F}\right)_{1 \leq i \leq k}$ is a $k$-symplectic manifold and $F$ becomes a symplectomorphism between $\left(T_{k}^{1} \mathbb{R}^{n},\left(\omega_{F}\right)_{i}\right)_{1 \leq i \leq k}$ and $\left(\left(T_{k}^{1}\right)^{*} \mathbb{R}^{n}, \omega_{i}\right)_{1 \leq i \leq k}$. Let us remark that the projection $\pi_{F}$ that defines the distribution $V_{F}$ is the pull-back by $F$ of the projection $\pi$ that defines the distribution $V$ [3]. Therefore, the two distributions $V$ and $V_{F}$ are connected by the relation $V=F_{*} V_{F}$.

Note that a diffeomorphism between $T_{k}^{1} \mathbb{R}^{n}$ and $\left(T_{k}^{1}\right)^{*} \mathbb{R}^{n}$ can be the Legendre transformation $T L$ associated to a regular Lagrangian $L \in C^{\infty}\left(T_{k}^{1} \mathbb{R}^{n}, \mathbb{R}\right)$, that is

$$
T L: T_{k}^{1} \mathbb{R}^{n} \rightarrow\left(T_{k}^{1}\right)^{*} \mathbb{R}^{n}
$$

defined by

$$
\left(T L\left(v_{1 q}, \ldots, v_{k q}\right)\right)^{i}\left(w_{q}\right):=\left.\frac{\mathrm{d}}{\mathrm{d} s}\right|_{s=0} L\left(v_{1 q}, \ldots, v_{i q}+s w_{q}, \ldots, v_{k q}\right), \quad 1 \leq i \leq k .
$$

Let $H \in C^{\infty}\left(\left(T_{k}^{1}\right)^{*} \mathbb{R}^{n}, \mathbb{R}\right)$.

Definition 1. [8] $X_{H}^{i}=\left(X_{1 H}^{i}, \ldots, X_{k H}^{i}\right), 1 \leq i \leq k$, are called the Hamiltonian vector fields on $\left(T_{k}^{1}\right)^{*} \mathbb{R}^{n}$ associated to the Hamiltonian $H$ if they are solutions of the Hamilton's equations

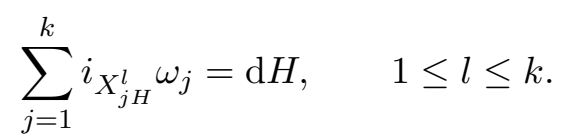

In Darboux coordinates $\left(q^{\alpha}, p_{\alpha}^{i}\right)_{1 \leq \alpha \leq n, 1 \leq i \leq k}$, the Hamiltonian vector fields are given by

$$
X_{j H}^{i}=\sum_{\alpha=1}^{n}\left(\frac{\partial H}{\partial p_{\alpha}^{j}} \cdot \frac{\partial}{\partial q^{\alpha}}-\delta_{j}^{i} \frac{\partial H}{\partial q^{\alpha}} \cdot \frac{\partial}{\partial p_{\alpha}^{j}}\right), \quad 1 \leq i, j \leq k .
$$


Proposition 2. ([8]) A Poisson structure on $\left(T_{k}^{1}\right)^{*} \mathbb{R}^{n}$ is given by

$$
\{f, h\}=\sum_{i=1}^{k} \omega_{i}\left(X_{i f}^{i}, X_{i h}^{i}\right)
$$

where $f, h \in C^{\infty}\left(\left(T_{k}^{1}\right)^{*} \mathbb{R}^{n}, \mathbb{R}\right)$ and $X_{f}^{i}=\left(X_{1 f}^{i}, \ldots, X_{k f}^{i}\right), X_{h}^{i}=\left(X_{1 h}^{i}, \ldots, X_{k h}^{i}\right)$, $1 \leq i \leq k$, are the corresponding Hamiltonian vector fields.

In [2] we have shown that

$$
\{f, h\}_{F}=F^{*}\left\{F^{*-1} f, F^{*-1} h\right\}
$$

$f, h \in C^{\infty}\left(T_{k}^{1} \mathbb{R}^{n}, \mathbb{R}\right)$, is a Poisson structure on $T_{k}^{1} \mathbb{R}^{n}$.

In order to get Poisson structures on $T_{k}^{1} \mathbb{R}^{n} / G$ and $\left(T_{k}^{1}\right)^{*} \mathbb{R}^{n} / G$, we need some additional assumptions concerning the actions of $G$ on these spaces. If the Lie group $G$ acts freely and properly on $T_{k}^{1} \mathbb{R}^{n}$ and $\left(T_{k}^{1}\right)^{*} \mathbb{R}^{n}$, then the quotient spaces are smooth manifolds. Moreover, assume that $G$ acts canonically on $T_{k}^{1} \mathbb{R}^{n}$ and $\left(T_{k}^{1}\right)^{*} \mathbb{R}^{n}$ respectively via the maps $\Phi_{g}^{T_{k}^{*}}$ and $\Phi_{g}^{T_{k}}$ respectively, that is

$$
\left(\Phi_{g}^{T_{k}}\right)^{*}\{f, h\}_{F}=\left\{\left(\Phi_{g}^{T_{k}}\right)^{*}(f),\left(\Phi_{g}^{T_{k}}\right)^{*}(h)\right\}_{F}, \quad g \in G
$$

$f, h \in C^{\infty}\left(T_{k}^{1} \mathbb{R}^{n}, \mathbb{R}\right)$ and

$$
\left(\Phi_{g}^{T_{k}^{*}}\right)^{*}\{f, h\}=\left\{\left(\Phi_{g}^{T_{k}^{*}}\right)^{*}(f),\left(\Phi_{g}^{T_{k}^{*}}\right)^{*}(h)\right\}, \quad g \in G
$$

$f, h \in C^{\infty}\left(\left(T_{k}^{1}\right)^{*} \mathbb{R}^{n}, \mathbb{R}\right)$.

Denote by $\pi:\left(T_{k}^{1}\right)^{*} \mathbb{R}^{n} \rightarrow \mathbb{R}^{n}, \pi\left(\alpha_{1 q}, \ldots, \alpha_{k q}\right):=q$, the canonical projection and assume that $\pi \circ F=\pi_{F}$. As $F$ is compatible with the equivalence relations that define the quotient manifolds $T_{k}^{1} \mathbb{R}^{n} / G$ and $\left(T_{k}^{1}\right)^{*} \mathbb{R}^{n} / G$ (that means, $F$ maps each orbit of the action of $G$ on $T_{k}^{1} \mathbb{R}^{n}$ onto an orbit of the action of $G$ on $\left.\left(T_{k}^{1}\right)^{*} \mathbb{R}^{n}\right)$, it induces a diffeomorphism $[F]: T_{k}^{1} \mathbb{R}^{n} / G \rightarrow\left(T_{k}^{1}\right)^{*} \mathbb{R}^{n} / G$ such that the following diagram commutes

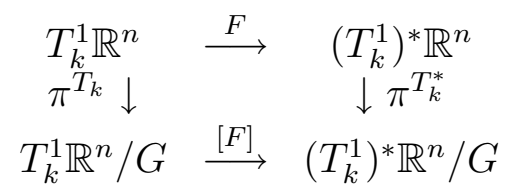

where $\pi^{T_{k}^{*}}:\left(T_{k}^{1}\right)^{*} \mathbb{R}^{n} \rightarrow\left(T_{k}^{1}\right)^{*} \mathbb{R}^{n} / G$ and $\pi^{T_{k}}: T_{k}^{1} \mathbb{R}^{n} \rightarrow T_{k}^{1} \mathbb{R}^{n} / G$ are the canonical projections. 
Under the hypotheses above and following [7], the reduced spaces $\left(T_{k}^{1}\right)^{*} \mathbb{R}^{n} / G$ and $T_{k}^{1} \mathbb{R}^{n} / G$ are Poisson manifolds, too, with the Poisson structures given by

$$
\{f, h\}^{\left(T_{k}^{1}\right)^{*} \mathbb{R}^{n} / G}\left(\pi^{T_{k}^{*}}\left(\alpha_{1}, \ldots, \alpha_{k}\right)\right):=\left\{f \circ \pi^{T_{k}^{*}}, h \circ \pi^{T_{k}^{*}}\right\}\left(\alpha_{1}, \ldots, \alpha_{k}\right)
$$

$f, h \in C^{\infty}\left(\left(T_{k}^{1}\right)^{*} \mathbb{R}^{n} / G, \mathbb{R}\right),\left(\alpha_{1}, \ldots, \alpha_{k}\right) \in\left(T_{k}^{1}\right)^{*} \mathbb{R}^{n}$ and respectively by

$$
\{f, h\}_{F}^{T_{k}^{1} \mathbb{R}^{n} / G}\left(\pi^{T_{k}}\left(v_{1}, \ldots, v_{k}\right)\right):=\left\{f \circ \pi^{T_{k}}, h \circ \pi^{T_{k}}\right\}_{F}\left(v_{1}, \ldots, v_{k}\right)
$$

$f, h \in C^{\infty}\left(T_{k}^{1} \mathbb{R}^{n} / G, \mathbb{R}\right),\left(v_{1}, \ldots, v_{k}\right) \in T_{k}^{1} \mathbb{R}^{n}$.

Then we have

Proposition 3. For any $f, h \in C^{\infty}\left(\left(T_{k}^{1}\right)^{*} \mathbb{R}^{n} / G, \mathbb{R}\right)$, the two reduced Poisson structures are connected by the relation

$$
[F]^{*}\{f, h\}^{\left(T_{k}^{1}\right)^{*} \mathbb{R}^{n} / G}=\left\{[F]^{*}(f),[F]^{*}(h)\right\}_{F}^{T_{k}^{1} \mathbb{R}^{n} / G}
$$

that is, $[F]$ is a Poisson diffeomorphism.

Proof: Since $F$ is a diffeomorphism compatible with the equivalence relations that define the quotient manifolds $\left(T_{k}^{1}\right)^{*} \mathbb{R}^{n} / G$ and $T_{k}^{1} \mathbb{R}^{n} / G$, for any $\left(v_{1}, \ldots, v_{k}\right) \in$ $T_{k}^{1} \mathbb{R}^{n}$ and for any $f, h \in C^{\infty}\left(\left(T_{k}^{1}\right)^{*} \mathbb{R}^{n} / G, \mathbb{R}\right)$, we obtain successively

$$
\begin{aligned}
& \left([F]^{*}\{f, h\}^{\left(T_{k}^{1}\right)^{*} \mathbb{R}^{n} / G}\right)\left(\pi^{T_{k}}\left(v_{1}, \ldots, v_{k}\right)\right) \\
& =\left(\{f, h\}^{\left(T_{k}^{1}\right)^{*} \mathbb{R}^{n} / G} \circ[F]\right)\left(\pi^{T_{k}}\left(v_{1}, \ldots, v_{k}\right)\right) \\
& =\{f, h\}^{\left(T_{k}^{1}\right) * \mathbb{R}^{n} / G}\left(\left([F] \circ \pi^{T_{k}}\right)\left(v_{1}, \ldots, v_{k}\right)\right) \\
& =\{f, h\}^{\left(T_{k}^{1}\right)^{*} \mathbb{R}^{n} / G}\left(\left(\pi^{\left.\left.T_{k}^{*} \circ F\right)\left(v_{1}, \ldots, v_{k}\right)\right)}\right.\right. \\
& =\left\{f \circ \pi^{T_{k}^{*}}, h \circ \pi^{T_{k}^{*}}\right\}\left(F\left(v_{1}, \ldots, v_{k}\right)\right) \\
& =\left(F^{*}\left\{f \circ \pi^{T_{k}^{*}}, h \circ \pi^{T_{k}^{*}}\right\}\right)\left(v_{1}, \ldots, v_{k}\right) \\
& =\left\{F^{*}\left(f \circ \pi^{T_{k}^{*}}\right), F^{*}\left(h \circ \pi^{T_{k}^{*}}\right)\right\}\left(v_{1}, \ldots, v_{k}\right) \\
& =\left\{f \circ\left(\pi^{T_{k}^{*}} \circ F\right), h \circ\left(\pi^{T_{k}^{*}} \circ F\right)\right\}\left(v_{1}, \ldots, v_{k}\right) \\
& =\left\{f \circ\left([F] \circ \pi^{T_{k}}\right), h \circ\left([F] \circ \pi^{T_{k}}\right)\right\}\left(v_{1}, \ldots, v_{k}\right) \\
& =\left\{[F]^{*}(f) \circ \pi^{T_{k}},[F]^{*}(h) \circ \pi^{T_{k}}\right\}\left(v_{1}, \ldots, v_{k}\right) \\
& =\left\{[F]^{*}(f),[F]^{*}(h)\right\}_{F}^{T_{k}^{1} \mathbb{R}^{n} / G}\left(\pi^{T_{k}}\left(v_{1}, \ldots, v_{k}\right)\right) \text {. }
\end{aligned}
$$


Remark 4. The reason for which we have chosen the Poisson brackets to be defined by the relations (9) and (10) is that they are suitable for the reduction of the Hamiltonian dynamics in the following sense: if $\pi: M \rightarrow M / G$ is the canonical projection, $H \in C^{\infty}(M, \mathbb{R})$ a $G$-invariant Hamiltonian on $M$, and

$$
\{f, h\}^{M / G}(\pi(x)):=\{f \circ \pi, h \circ \pi\}(x)
$$

$f, h \in C^{\infty}(M / G, \mathbb{R}), x \in M$, the Poisson bracket on $M / G$, then the Hamiltonian flow $F_{t}$ of the Hamiltonian vector field $X_{H}$ commutes with the G-action. Thus it induces the flow $F_{t}^{M / G}$ on $G / M$ associated to the reduced Hamiltonian $[H] \in C^{\infty}(M / G, \mathbb{R})$, where $[H] \circ \pi=H$.

\section{Acknowledgements}

I wish to express my thanks to the referees for the useful remarks.

\section{References}

[1] Awane A., k-symplectic Structures, J. Math. Phys. 33 (1992) 4046-4052.

[2] Blaga A., The Prequantization of $T_{k}^{1} \mathbb{R}^{n}$, In: Differential Geometry and Its Applications, World Scientific 2008, pp. 217-222, Proceedings of the 10th International Conference on Differential Geometry and Its Applications, Olomouc, Czech Republic, 2007.

[3] De Leon M., Merino E., Qubiña J., Rodrigues P. and Salgado M., Hamiltonian Systems on k-cosymplectic Manifolds, J. Math. Phys. 39 (1998) 876893.

[4] Marsden J. and Weinstein A., The Hamiltonian Structure of the MaxwellVlasov Equations, Physica D4 (1982) 394-406.

[5] Marsden J. and Weinstein A., Coadjoint Orbits, Vortices and Clebsch Variables for Incompressible Fluids, Physica D4 (1983) 305-323.

[6] Munteanu F., Rey A. and Salgado M., The Günther's Formalism in Classical Field Theory: Momentum Map and Reduction, J. Math. Phys. 45 (2004) 1730-1751.

[7] Ortega J. and Raţiu T., Poisson Reduction, arXiv: math. SG / 0508635 v1 2005.

[8] Puta M., Chirici S. and Merino E., On the Prequantization of $\left(T_{k}^{1}\right)^{*} \mathbb{R}^{n}$, Bull. Math. Soc. Sc. Math. Roumanie 44 (2001) 277-284. 
Adara M. Blaga

Department of Mathematics andInformatics

West University of Timisoara

Bld. V. Parvan nr. 4

Timisoara 300223

ROMANIA

E-mail address: adara@math.uvt.ro 